\title{
Clinical Significance of Preoperative Fibrinogen to Albumin Ratio in Patients with Glioblastoma: A Singe Center Experience
}

This article was published in the following Dove Press journal: Cancer Management and Research

\section{Junhong $\mathrm{Li}$ (D) \\ Xingwang Zhou \\ Yufan Xiang \\ Shuxin Zhang \\ Wentao Feng \\ Yunbo Yuan \\ Yanhui Liu \\ Senlin Yin}

Department of Neurosurgery, West China Hospital of Sichuan University, Chengdu, Sichuan Province, 6I004I,

People's Republic of China
Correspondence: Senlin Yin Email enforest@gmail.com
Objective: To explore the prognostic value of preoperative fibrinogen to albumin ratio (FAR) in patients with glioblastoma (GBM) and its association with clinical characteristics. Patients and Methods: A retrospective analysis was carried out on patients with newly diagnosed GBM who had undergone operation at the Department of Neurosurgery at West China Hospital between June 1st 2015 to June 31st 2018. Receiver operating characteristic (ROC) curves were performed to determine the optimal cut-off values for fibrinogen, albumin, neutrophil to lymphocyte ratio (NLR), and FAR by calculating the maximum Youden index. Kaplan-Meier curves and Cox regression analyses were applied to evaluate the prognostic value of FAR in GBM. Harrell concordance index (C-index) and Akaike information criterion (AIC) were calculated to compare different prognostic models.

Results: A total of 206 GBM patients were included in this research. The optimal cut-off value for fibrinogen, albumin, NLR, and FAR were 2.57, 42.4, 2.28, and 0.068 respectively. High FAR was significantly related to older age, KPS $\leq 80$, IDH-1 wildtype, presence of preoperative seizures, higher NLR, and tumor location. In Cox regression analyses, high FAR was significantly associated with poor prognosis. Prognostic models including FAR had the largest C-index and lowest AIC.

Conclusion: FAR was determined to be an independent risk factor of prognosis in patients with newly-diagnosed GBM. And the prognostic predictive ability of FAR is stronger than fibrinogen and albumin.

Keywords: fibrinogen to albumin ratio, FAR, fibrinogen, albumin, glioblastoma, prognosis

\section{Introduction}

Glioblastoma (GBM), which is the most common and lethal primary malignancy in central nervous system, is characterized by tumor necrosis, microvascular proliferation, and elevated tumor cell proliferation. ${ }^{1}$ Median overall survival is 15-23 months and 5-year survival rate is less than $6 \%$ even though those receiving standard treatments including surgery, radiotherapy, and chemotherapy. ${ }^{2}$ Approximately 90\% GBMs primarily occur in elderly patients, whereas in younger patients, it usually progresses from lower grade astrocytomas. ${ }^{3}$ Based on the newly 2016 World Health Organization Classification of Tumors, isocitrate dehydrogenase-1 (IDH-1) was formally introduced into the differentiation and diagnosis of GBM, which initiated new era of molecular diagnosis of GBM. ${ }^{4,5}$ It remains unknown about the molecular mechanisms how GBM develops so that specific therapeutic targets cannot be easily determined. In recent years, it has been an 
eternal topic in the field of cancer to find effective biomarkers to predict and treat GBM.

Previous laboratory tests have found that the process of fibrin formation and fibrinolysis parallels the progress of malignancy. Hemostatic system including thrombin, tissue factor and fibrinogen has been verified playing an important role in neoangiogenesis and metastasis of different tumors. ${ }^{6}$ Fibrinogen, also known as coagulation factor I, is a $340 \mathrm{kDa}$ glycoprotein synthesized in the liver. As a pro-inflammatory protein, it has been reported in connection with progression and prognosis of tumors, such as upper urinary tract urothelial carcinoma, gastrointestinal stromal tumors, pancreatic cancer, and epithelial ovarian cancer. $^{7-10}$ Similarly, produced by the liver, plasma albumin plays a regulatory role in plasma oncotic pressure, solubilization, antioxidantion, immunomodulation, and capillary permeability, etc. ${ }^{11,12}$ It is also widely used as an indicator of nutritional status. Recent studies have indicated an association between plasma albumin concentration, inflammation and tumorigenesis. ${ }^{13,14}$

Systemic inflammation response markers are represented by neutrophil to lymphocyte ratio (NLR), plateletlymphocyte ratio (PLR), albumin to globulin ratio (AGR) and immune-inflammation index, and as one member of which, fibrinogen to albumin ratio (FAR) is a novel indicator of prognosis in various tumors. ${ }^{15-17}$ To our knowledge, there are no relevant researches related to the clinical significance of FAR in patients with GBM. Therefore, this study is conducted to explore the potential relationship between FAR and GBM.

\section{Patients and Methods}

\section{Patient Population}

A retrospective chart review was carried out on patients with newly diagnosed GBM who had undergone an operation at the Department of Neurosurgery at West China Hospital between June 1st 2015 to June 31st 2018. All patients underwent a craniotomy on GBM with total resection or subtotal resection. These patients were followed up until June 31st 2020. The pathological diagnoses were based on 2016 WHO classification of CNS tumors. The exclusion criteria were: 1) biopsy only; 2) younger than 18-year-old; 3) absence of definite pathological diagnosis; 4) incomplete baseline clinical data; 5) absence of preoperative MRI imaging data; 6) receiving adjuvant therapy like chemotherapy or radiotherapy before operation; 7) presence of history of liver diseases, infectious diseases, blood system diseases or in a low nutrition condition shortly before surgery.

\section{Parameters Assessment}

Medical records were surveyed and the following clinical data were retrieved: gender, age at operation, preoperative Karnofsky performance status, presence of preoperative seizures, locations and hemisphere of tumors, pathological diagnoses and some biomarkers. Ki-67 index was tested by immunohistochemistry (IHC), while IDH-1 mutations were determined by both IHC and molecular testing. Routine blood tests were performed within 3 days prior to surgery and relevant data was recorded. The FAR was defined as the ratio between the serum fibrinogen concentration $(\mathrm{g} / \mathrm{L})$ and the albumin $(\mathrm{g} / \mathrm{L})$, while the NLR was defined by dividing the neutrophil $\left(\times 10^{9} / \mathrm{L}\right)$ count by the lymphocyte count $\left(\times 10^{9} / \mathrm{L}\right)$.

Postoperative adjuvant therapies and survival conditions were collected mainly through periodical telephone interview and outpatient follow-up. Patients were routinely followed up every 3 months for the first year, and every 6 months thereafter. Overall survival was defined as the duration from the date of surgery to death or the last follow up. All clinical assessments were performed by two independent qualified neurosurgeons.

\section{Statistical Analysis}

SPSS 22.0 (IBM Co., Armonk, NY, USA) was used for all statistical analyses. Receiver operating characteristic (ROC) curves were performed to determine the optimal cut-off values by calculating the maximum Youden index. The associations between APPR and clinical variables were tested by chi-square test, Mann-Whitney test, or oneway ANOVA (one-factor analysis of variance). The Kaplan-Meier (K-M) curves and Cox regression analyses were used to determine the influence of risk factors for overall survival in GBM patients. In terms of Cox regression analyses, a univariate Cox regression was firstly conducted to evaluate clinical variables, then variables with $\mathrm{p}$ value $<0.1$ were included into a backward stepwise multivariate Cox regression for further assessment. $\mathrm{R}$ software (version 3.6.3, http://www.r-project.org/) was applied to calculate and compare Harrell concordance index (C-index) and Akaike information criterion (AIC) of different prognostic models. A smaller AIC value and/or a larger C-index represented a greater predictive accuracy. A two-sided p-value $<0.05$ referred as statistically significant difference. 


\section{Ethics}

This study was approved by the Ethical Committee of Sichuan University and conducted according to the principles expressed in the Declaration of Helsinki, and all patients were informed and signed their informed consent to use their data for research purposes.

\section{Results}

\section{Baseline Characteristics}

The research group was constituted by a total of 206 consecutive patients with craniotomy for histologicallyproven glioblastoma (Table 1). There were 133 (65.56\%) males and 73 (35.44\%) females, with a mean age of 54.25 \pm 0.90 years (median 55 , range $20-81$ years). The mean follow-up period was $467.08 \pm 24.60$ days (median 353, range 31-1611 days). In terms of tumor-related seizures, $37(17.96 \%)$ patients were diagnosed with preoperative seizures. A preoperative KPS score $>80$ was recorded in $74(35.92 \%)$ patients and vice versa. For location of GBMs, 99 GBMs (48.06\%) were located at left hemisphere, $94(45.63 \%)$ at right hemisphere, and $13(6.31 \%)$ at midline regions or invading bilateral brain tissues. These tumors were distributed in frontal lobe (25.24\%), temporal lobe $(19.42 \%)$, parietal lobe $(6.80 \%)$, occipital lobe $(4.37 \%)$, and insular lobe $(7.77 \%)$, and the rest were involved in multiple regions $(36.40 \%)$.

As for postoperative adjuvant therapy, 71 (34.47\%) patients received both chemotherapy and radiotherapy, 62 $(30.10 \%)$ patients received one of the two treatments, while other $73(35.43 \%)$ patients did not receive any kind of adjuvant therapy due to various reasons. Two glioma-related biomarkers were recorded; a total of 111 $(53.88 \%)$ patients had ki-67 index $<30 \%$, while 29 (14.08\%) patients were tested as IDH-1 mutation.

According to ROC curves (Figure 1), the optimal cutoff value for serum fibrinogen and albumin were $2.57 \mathrm{~g} / \mathrm{L}$ and $42.4 \mathrm{~g} / \mathrm{L}$, with the area under the curve (AUC) of $0.609(0.517-0.701, \mathrm{p}=0.022)$ and $0.618(0.525-0.712$, $\mathrm{p}=0.013)$, respectively. The optimal cut-off value for FAR and NLR were 0.068 and 2.28, with the AUC of $0.626(0.533-0.720, \mathrm{p}=0.008)$ and $0.586(0.508-0.664$, $\mathrm{p}=0.033)$, respectively.

\section{Relationships Between FAR and Clinical Variables}

The relationships between FAR and other clinical variables were shown in Table 2. High FAR was significantly related
Table I Baseline Clinical Characteristics of 206 Patients with Glioblastoma

\begin{tabular}{|c|c|c|}
\hline \multicolumn{2}{|c|}{ Clinical Characteristics } & \multirow{2}{*}{$\begin{array}{c}\text { Value (\%) } \\
206(100 \%)\end{array}$} \\
\hline Sample Size & & \\
\hline \multirow[t]{2}{*}{ Follow-up period } & Mean \pm SD (day) & $467.08 \pm 24.60$ \\
\hline & Median(range) & $353(3 I-16 I I)$ \\
\hline \multirow[t]{2}{*}{ Age at operation } & Mean \pm SD (year) & $54.25 \pm 0.90$ \\
\hline & Median(range) & $55(20-81)$ \\
\hline \multirow[t]{2}{*}{ Gender } & Male & I 33 (64.56\%) \\
\hline & Female & 73 (35.44\%) \\
\hline \multirow[t]{2}{*}{ Preoperative seizures } & Yes & 169 (82.04\%) \\
\hline & No & 37 (17.96\%) \\
\hline \multirow{2}{*}{$\begin{array}{l}\text { Karnofsky } \\
\text { performance status }\end{array}$} & $>80$ & 74 (35.92\%) \\
\hline & $\leq 80$ & I 32 (64.08\%) \\
\hline \multirow[t]{3}{*}{ Hemisphere } & Left & $99(48.06 \%)$ \\
\hline & Right & $94(45.63 \%)$ \\
\hline & Midline or bilateral & $13(6.31 \%)$ \\
\hline \multirow[t]{6}{*}{ Location } & Frontal lobe & $52(25.24 \%)$ \\
\hline & Temporal lobe & 40 (19.42\%) \\
\hline & Parietal lobe & $14(6.80 \%)$ \\
\hline & Occipital lobe & $9(4.37 \%)$ \\
\hline & Insular lobe & $16(7.77 \%)$ \\
\hline & Other locations & 75 (36.40\%) \\
\hline \multirow[t]{3}{*}{ Adjuvant therapy } & $\begin{array}{l}\text { Chemotherapy and } \\
\text { radiotherapy }\end{array}$ & 71 (34.47\%) \\
\hline & Chemotherapy or & $62(30.10 \%)$ \\
\hline & None & 73 (35.43\%) \\
\hline \multirow[t]{2}{*}{$\mathrm{Ki}-67$} & $\geq 30 \%$ & $95(46.12 \%)$ \\
\hline & $<30 \%$ & III (53.88\%) \\
\hline \multirow[t]{2}{*}{ IDH-I } & Mutation & $29(14.08 \%)$ \\
\hline & Wildtype & I 77 (85.92\%) \\
\hline \multirow[t]{2}{*}{ Fibrinogen (g/L) } & $\geq 2.57$ & 120 (58.25\%) \\
\hline & $<2.57$ & 86 (4I.75\%) \\
\hline \multirow[t]{2}{*}{ Albumin $(g / L)$} & $\geq 42.4$ & 105 (50.97\%) \\
\hline & $<42.4$ & I0I (49.03\%) \\
\hline \multirow[t]{2}{*}{ FAR } & $\geq 0.068$ & 87 (42.23\%) \\
\hline & $<0.068$ & 119 (57.77\%) \\
\hline \multirow[t]{2}{*}{ NLR } & $\geq 2.28$ & 127 (6I.65\%) \\
\hline & $<2.28$ & 79 (38.35\%) \\
\hline
\end{tabular}

Abbreviations: IDH-I, isocitrate dehydrogenase-I; NLR, neutrophil to lymphocyte ratio; FAR, fibrinogen to albumin ratio.

to variables including older age $(\mathrm{p}<0.001), \mathrm{KPS} \leq 80$ $(\mathrm{p}<0.001)$, IDH-1 wildtype $(\mathrm{p}=0.041)$, and presence of preoperative seizures $(p=0.021)$. Similarly, patients with $\mathrm{NLR} \geq 2.28$ had a higher FAR $(\mathrm{p}<0.001)$. As regards to 

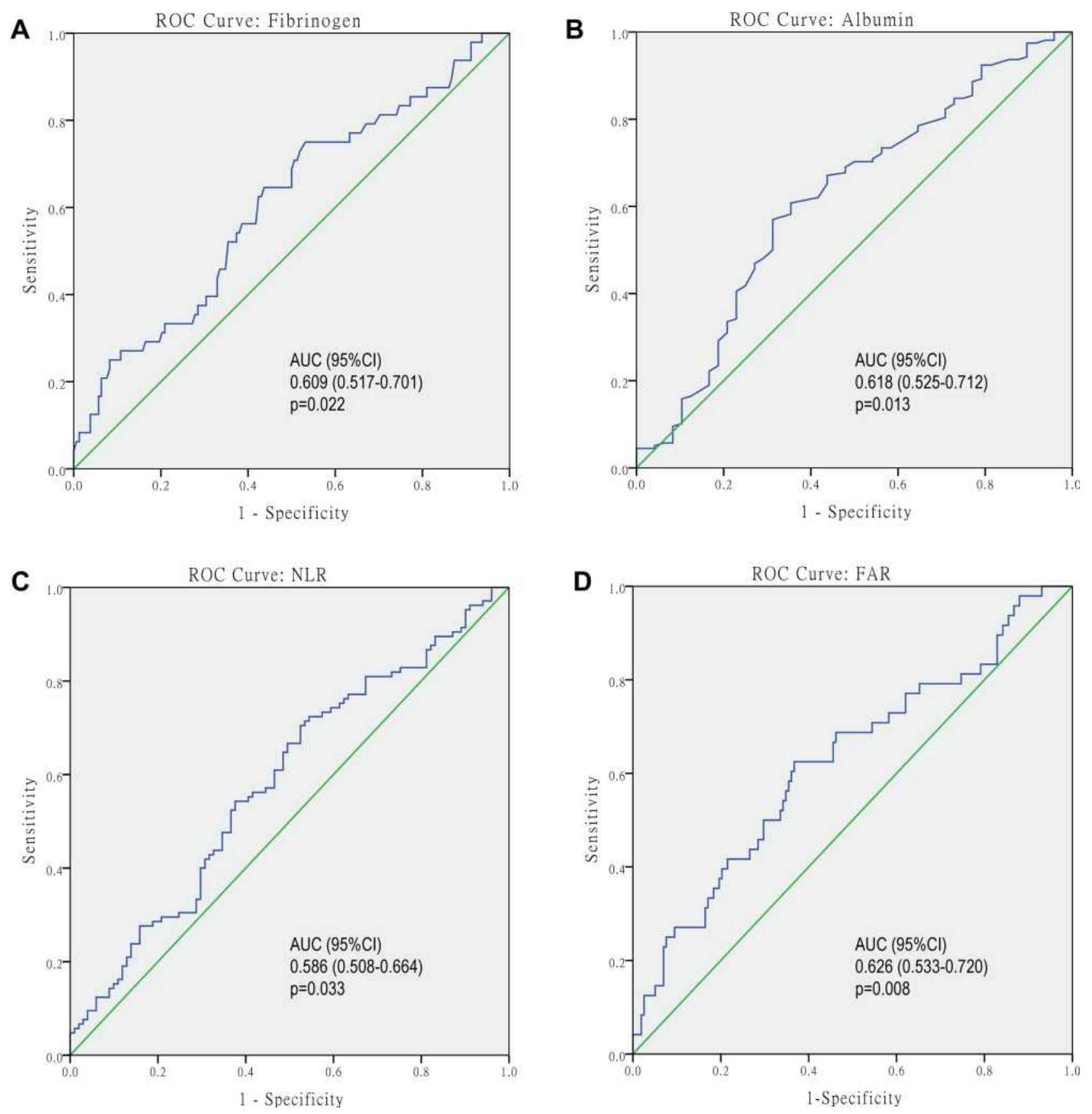

Figure I Receiver operating characteristic (ROC) analyses were performed to evaluate the optimal cut-off values of fibrinogen (A), albumin (B), NLR (C), and FAR (D), and cut-off values were determined using the Youden index.

Abbreviations: NLR, neutrophil to lymphocyte ratio; FAR, fibrinogen to albumin ratio.

tumor location, tumor located at temporal lobe and occipital lobe seemed to have high FAR ( $\mathrm{p}=0.043$ ).

There was no significant association between FAR and other clinical variables including gender, hemisphere and Ki-67 index.

\section{Prognostic Value of FAR}

As shown in Figure 2, K-M curves indicated that patients with lower FAR had longer OS, compared to those with higher FAR ( $\log$ rank test, $\mathrm{p}<0.001$ ). Patients with lower fibrinogen and NLR had a better OS (Log rank test, 
Table 2 Relationship Between FAR and Clinical Characteristics

\begin{tabular}{|c|c|c|c|c|c|}
\hline \multicolumn{2}{|c|}{ Clinical Characteristics } & \multirow{2}{*}{$\begin{array}{l}\text { FAR }<0.068 \\
(n=119)\end{array}$} & \multirow{2}{*}{$\begin{array}{c}\text { FAR } \geq 0.068 \\
(n=87)\end{array}$} & \multirow{2}{*}{$\begin{array}{c}\text { FAR } \\
\text { (Mean } \pm S D)\end{array}$} & \multirow[t]{2}{*}{$P$ value } \\
\hline & & & & & \\
\hline Age at operation & $\begin{array}{l}\geq 55 \\
<55\end{array}$ & $\begin{array}{l}46 \\
73\end{array}$ & $\begin{array}{l}58 \\
29\end{array}$ & $\begin{array}{l}0.062 \pm 0.002 \\
0.079 \pm 0.003\end{array}$ & $<0.001$ \\
\hline Gender & $\begin{array}{l}\text { Male } \\
\text { Female }\end{array}$ & $\begin{array}{l}69 \\
50\end{array}$ & $\begin{array}{l}64 \\
23\end{array}$ & $\begin{array}{l}0.073 \pm 0.003 \\
0.065 \pm 0.002\end{array}$ & 0.098 \\
\hline Preoperative seizures & $\begin{array}{l}\text { Yes } \\
\text { No }\end{array}$ & $\begin{array}{l}26 \\
93\end{array}$ & $\begin{array}{l}11 \\
76\end{array}$ & $\begin{array}{l}0.063 \pm 0.004 \\
0.072 \pm 0.002\end{array}$ & 0.021 \\
\hline KPS & $\begin{array}{l}>80 \\
\leq 80\end{array}$ & $\begin{array}{l}57 \\
62\end{array}$ & $\begin{array}{l}17 \\
70\end{array}$ & $\begin{array}{l}0.060 \pm 0.002 \\
0.076 \pm 0.003\end{array}$ & $<0.001$ \\
\hline Hemisphere & $\begin{array}{l}\text { Left } \\
\text { Right } \\
\text { Midline or bilateral }\end{array}$ & $\begin{array}{l}66 \\
46 \\
7\end{array}$ & $\begin{array}{l}33 \\
48 \\
6\end{array}$ & $\begin{array}{l}0.067 \pm 0.003 \\
0.074 \pm 0.003 \\
0.066 \pm 0.005\end{array}$ & 0.129 \\
\hline Location & $\begin{array}{l}\text { Frontal lobe } \\
\text { Temporal lobe } \\
\text { Parietal lobe } \\
\text { Occipital lobe } \\
\text { Insular lobe } \\
\text { Other regions }\end{array}$ & $\begin{array}{l}33 \\
16 \\
9 \\
4 \\
10 \\
47\end{array}$ & $\begin{array}{l}19 \\
24 \\
5 \\
5 \\
6 \\
28\end{array}$ & $\begin{array}{l}0.068 \pm 0.004 \\
0.081 \pm 0.006 \\
0.064 \pm 0.003 \\
0.082 \pm 0.008 \\
0.065 \pm 0.005 \\
0.067 \pm 0.003\end{array}$ & 0.043 \\
\hline Ki-67 & $\begin{array}{l}\geq 30 \% \\
<30 \%\end{array}$ & $\begin{array}{l}54 \\
65\end{array}$ & $\begin{array}{l}41 \\
46\end{array}$ & $\begin{array}{l}0.073 \pm 0.004 \\
0.068 \pm 0.002\end{array}$ & 0.653 \\
\hline IDH-I & $\begin{array}{l}\text { Mutation } \\
\text { Wildtype }\end{array}$ & $\begin{array}{l}20 \\
99\end{array}$ & $\begin{array}{l}9 \\
78\end{array}$ & $\begin{array}{l}0.061 \pm 0.003 \\
0.072 \pm 0.002\end{array}$ & 0.041 \\
\hline NLR & $\begin{array}{l}\geq 2.28 \\
<2.28\end{array}$ & $\begin{array}{l}61 \\
58\end{array}$ & $\begin{array}{l}66 \\
21\end{array}$ & $\begin{array}{l}0.077 \pm 0.003 \\
0.060 \pm 0.002\end{array}$ & $<0.001$ \\
\hline
\end{tabular}

Abbreviations: IDH-I, isocitrate dehydrogenase-I; NLR, neutrophil to lymphocyte ratio; FAR, fibrinogen to albumin ratio.

$\mathrm{p}=0.005$ and $\mathrm{p}=0.036$, respectively). Rather, high albumin concentration predicted good prognosis $(\mathrm{p}=0.003)$.

Univariate and multivariate Cox regressions were performed to further determine the prognostic significance of FAR and other variables (Table 3). Univariate Cox regression suggested a significant association between high FAR (HR $1.778,95 \%$ CI 1.321-2.392, $\mathrm{p}<0.001$ ) short OS. And more over, other significant variables were age, gender, hemisphere, adjuvant therapy, IDH-1 status, NLR, albumin and fibrinogen.

Variables with a $\mathrm{p}$ value $<0.1$ were included in multivariate Cox regression. FAR was proved to be an independent risk factor of OS (HR=1.793, 95\% CI 1.312-2.451, $\mathrm{p}<0.001$ ). Concentration of fibrinogen and albumin were also determined to be independent predictors of OS $(\mathrm{HR}=1.738, \quad 95 \% \quad \mathrm{CI} \quad 1.266-2.385, \quad \mathrm{p}=0.001 \quad$ and $\mathrm{HR}=0.651,95 \%$ CI $0.477-0.888, \mathrm{p}=0.007$ respectively). Other independent risk factors included gender, adjuvant therapy, ki-67 index, and IDH-1 mutation status.

\section{Comparison of Prognostic Ability Between FAR, Fibrinogen and Albumin}

To further compare the prognostic predictive accuracy of the three chosen markers, prognostic models were established by using the three markers combined with other independent variables including gender, adjuvant therapy, ki-67 index, and IDH-1 mutation status. (Table 4) C-index and AIC were calculated by $\mathrm{R}$ and the results indicated that model FAR (C-index/AIC, 0.714/1576.170) had the optimal prognostic ability among the three candidates, which was superior to model albumin (C-index/AIC, $0.711 / 1583.814$ ) and model fibrinogen (C-index/AIC, $0.712 / 1576.516)$.

\section{Discussions}

In the current study, FAR is found not only significantly associated with some clinical characteristics, but also serving as an independent prognostic indicator in patients 

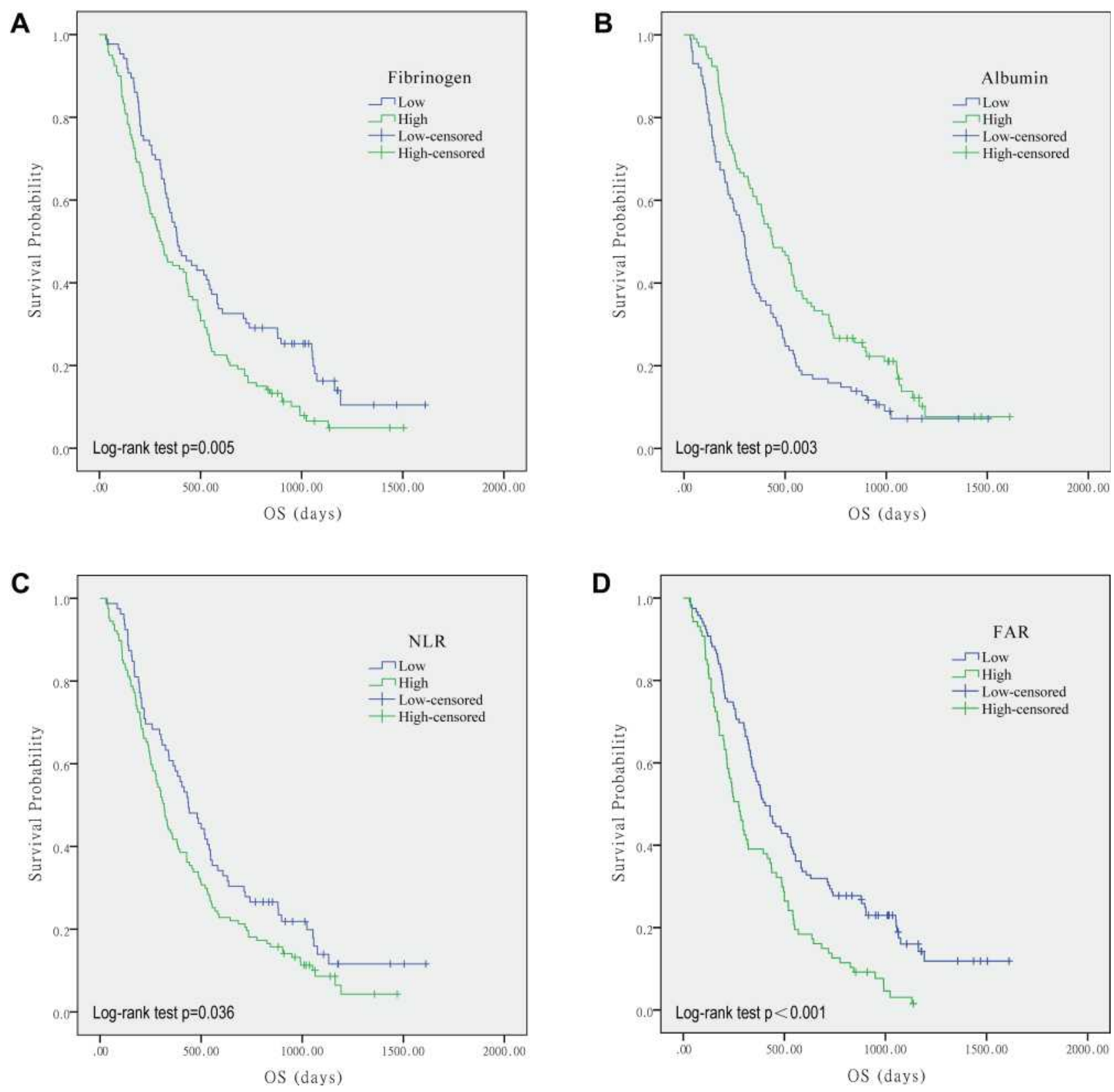

Figure 2 Kaplan-Meier curves showing overall survival of GBM patients stratified by value of fibrinogen (A), albumin (B), NLR (C), and FAR (D). Abbreviations: NLR, neutrophil to lymphocyte ratio; FAR, fibrinogen to albumin ratio.

with GBM. Although fibrinogen and albumin are also determined to be independent risk factors for OS, the prognostic ability is weaker than FAR.

FAR is a novel hematological marker, which is mainly used to predict prognosis in various tumors. In some researches, albumin to fibrinogen ratio (AFR) is preferred to FAR. Higher FAR predicts poor prognosis in most researches, but several studies indicated valid prognostic ability of FAR or AFR. In the retrospective research of Sun et al, stage II-III colorectal cancer patients are concerned and AFR is determined to be an independent prognostic indicator with an adjusted HR 0.709 (0.455-1.107). ${ }^{18}$ Similarly, Du et al study indicates that AFR is not an independent indicator for OS in patients with metastatic gallbladder cancer. ${ }^{19}$ The results are relatively suspectable in consideration of the fact that patients in specific stage or condition are included in these study. In previous study concerning glioma and FAR or AFR, we only find a research between grade III gliomas and FAR through 


\begin{tabular}{|c|c|c|c|c|c|c|c|c|c|c|c|c|}
\hline \multirow{4}{*}{ 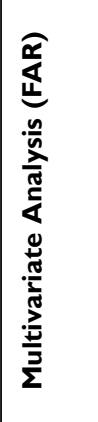 } & \multicolumn{2}{|l|}{$\begin{array}{l}\frac{0}{\underline{J}} \\
\frac{\pi}{2} \\
0\end{array}$} & 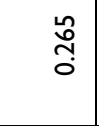 & ợ & 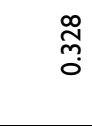 & $\begin{array}{ll} \pm \\
0\end{array}$ & & & & $\begin{array}{ll}\bar{\delta} & \bar{c} \\
\dot{\mathrm{v}} & \mathrm{c}\end{array}$ & $\overline{0}$ & 웅 \\
\hline & \multirow{2}{*}{$\begin{array}{l}\bar{u} \\
\text { نั่ } \\
\text { نे }\end{array}$} & 5 & 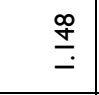 & 号 & 亦 & 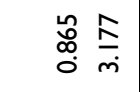 & & & & 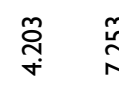 & 吕 & in \\
\hline & & 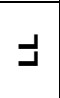 & $\begin{array}{l} \\
\\
\end{array}$ & \begin{tabular}{l} 
t. \\
\multirow{0}{0}{} \\
0
\end{tabular} & 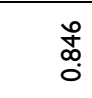 & 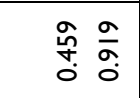 & & & & å & হ̦. & $\stackrel{m}{\tilde{q}}$ \\
\hline & \multicolumn{2}{|l|}{$\frac{\mathscr{x}}{I}$} & 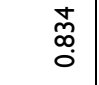 & 侽 & $\stackrel{\bar{\infty}}{=}$ & $\begin{array}{ll}\stackrel{0}{0} & \stackrel{0}{0} \\
0 & \stackrel{1}{-}\end{array}$ & & & & 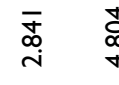 & 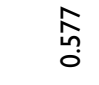 & $\underset{d}{\mathrm{~N}}$ \\
\hline \multirow{4}{*}{ 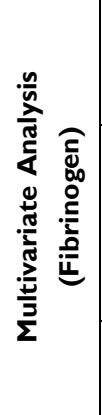 } & \multicolumn{2}{|l|}{$\begin{array}{l}\frac{0}{\mathcal{J}} \\
\frac{\pi}{2} \\
0\end{array}$} & 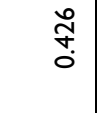 & రั & $\frac{\circ}{\circ}$ & 웅 & & & & $\begin{array}{ll}\bar{\delta} & \bar{c} \\
\dot{0} & \bar{c}\end{array}$ & $\overline{0}$ & ষे \\
\hline & \multirow{2}{*}{$\begin{array}{l}\bar{u} \\
\text { iें }\end{array}$} & 5 & $\overline{\bar{\Upsilon}}$ & $\frac{\infty}{\infty}$ & $\stackrel{\text { ș }}{=}$ & 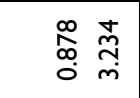 & & & & 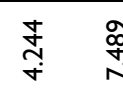 & $\begin{array}{l}0 \\
\substack{\infty \\
0 \\
0}\end{array}$ & $\stackrel{\substack{\infty \\
m}}{\sim}$ \\
\hline & & ل & 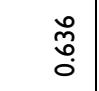 & 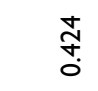 & $\frac{\infty}{a}$ & $\begin{array}{ll}\hat{f} & \bar{\sigma} \\
\text { ț. } & 0 \\
0 & 0\end{array}$ & & & & $\stackrel{\infty}{\stackrel{\infty}{*}} \stackrel{+}{\stackrel{N}{m}}$ & $\underset{\tilde{c}}{\tilde{0}}$ & 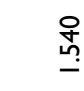 \\
\hline & \multicolumn{2}{|l|}{$\frac{\mathscr{I}}{\underline{I}}$} & $-\underset{\substack{\hat{\infty} \\
0}}{\mathbf{c}}$ & $-\underset{\substack{\infty \\
0}}{0}$ & $-\stackrel{\circ}{\stackrel{\circ}{!}}$ & 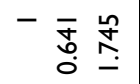 & & & - & 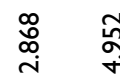 & - & - \\
\hline \multirow{4}{*}{ 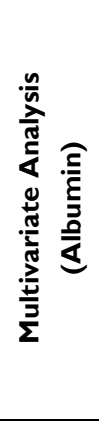 } & \multicolumn{2}{|l|}{$\begin{array}{l}\frac{0}{2} \\
\frac{N}{2} \\
0\end{array}$} & 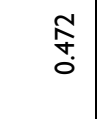 & 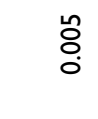 & 今̊ & $\bar{\delta} \frac{\circ}{0}$ & & & & $\begin{array}{ll}\overline{8} & \overline{0} \\
\dot{0} & \overline{0} \\
\mathrm{v} & \mathrm{v}\end{array}$ & ธุ & $\begin{array}{l}\bar{\Delta} \\
\text { v }\end{array}$ \\
\hline & \multirow{2}{*}{$\begin{array}{l}\bar{~} \\
\text { ٌั้ }\end{array}$} & 5 & $\underset{\sim}{\stackrel{m}{\longrightarrow}}$ & 灾 & $\underset{\substack{\infty \\
\stackrel{\infty}{\infty}}}{.}$ & 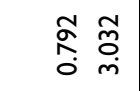 & & & & $\underset{f}{\frac{0}{f}} \quad \frac{\infty}{b}$ & $\underset{\substack{m \\
\infty \\
0}}{0}$ & 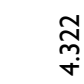 \\
\hline & & $\sqsupset$ & 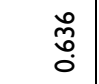 & $\stackrel{n}{\text { ִq }}$ & 옹 & 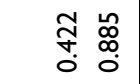 & & & & 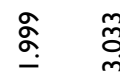 & $\underset{j}{+}$ & $\underline{\underline{n}}$ \\
\hline & 哇 & & \begin{tabular}{r|}
$-\underset{\infty}{\infty}$ \\
$\circ$ \\
\end{tabular} & 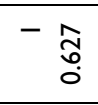 & 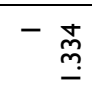 & $-\underset{c}{\infty} \underset{\hat{n}}{0} \stackrel{0}{0}$ & & & - & 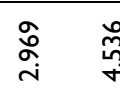 & $\begin{array}{r}-\infty \\
0 \\
0 \\
0\end{array}$ & 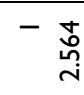 \\
\hline \multirow{4}{*}{ 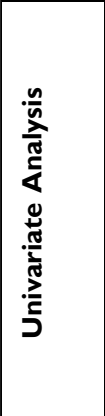 } & \multicolumn{2}{|l|}{$\begin{array}{l}\frac{0}{2} \\
\frac{2}{2} \\
2\end{array}$} & $\frac{0}{0}$ & $\hat{o}$ & 号 & 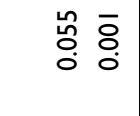 & 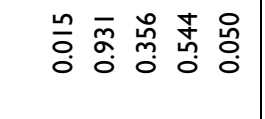 & $\frac{0}{m}$ & & $\begin{array}{l}\bar{a} \\
\text { v }\end{array}$ & 웅 & ষ্̀ \\
\hline & \multirow{2}{*}{$\begin{array}{l}\bar{u} \\
\text { แั่ }\end{array}$} & 5 & mà & $\begin{array}{l}\infty \\
\substack{\infty \\
0 \\
0}\end{array}$ & 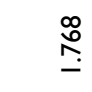 & $\underline{\underline{\delta}} \frac{m}{\dot{m}}$ & 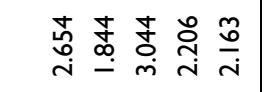 & $\stackrel{\bar{n}}{\underline{n}}$ & & $\begin{array}{ll}\stackrel{\infty}{\infty} & \infty \\
\stackrel{m}{m} & \stackrel{N}{\sigma}\end{array}$ & $\begin{array}{l}\stackrel{\circ}{\circ} \\
\stackrel{0}{\circ}\end{array}$ & 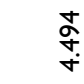 \\
\hline & & \lrcorner & 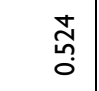 & f̊. & 峑 & 员 串 & 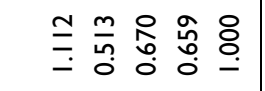 & $\underset{0}{\substack{\infty \\
0 \\
0}}$ & & 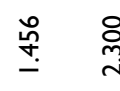 & 虽 & $\stackrel{\infty}{0}$ \\
\hline & $\frac{\mathscr{x}}{\mathbf{I}}$ & & 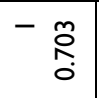 & $-\bar{n}$ & 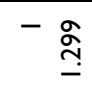 & $-\underset{0}{i} \frac{i}{0} \underset{i}{0}$ & 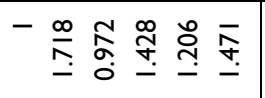 & $-\overline{\bar{N}}$ & - & $\frac{\infty}{\bar{N}} \stackrel{\infty}{m}$ & $-\underset{\substack{\stackrel{0}{0} \\
0}}{0}$ & $-\underset{i}{\stackrel{m}{i}}$ \\
\hline \multirow[b]{2}{*}{ 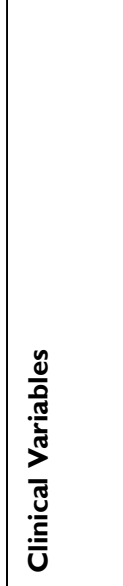 } & & & 㞻 苫 & $\frac{0}{\frac{0}{\pi}}$ & $\begin{array}{ll}\stackrel{\circ}{\circ} \\
\wedge \\
\wedge\end{array}$ & 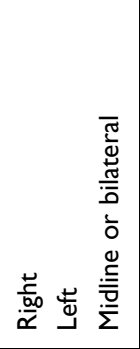 & 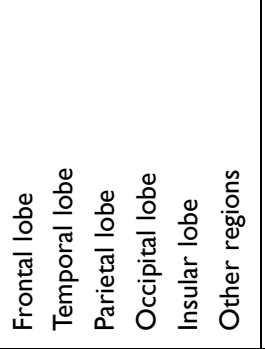 & $2 \stackrel{0}{z}$ & 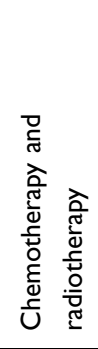 & 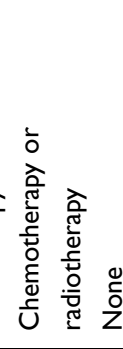 & 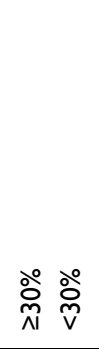 & 崖 \\
\hline & & & 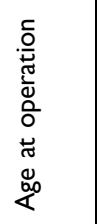 & 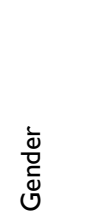 & 号 & 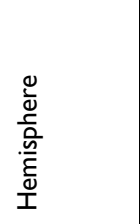 & 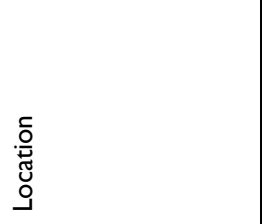 & 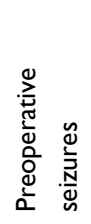 & 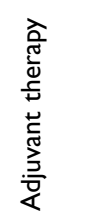 & & $\widehat{\widehat{o}}$ & $\begin{array}{l}\overline{\underline{I}} \\
\underline{\underline{I}}\end{array}$ \\
\hline
\end{tabular}




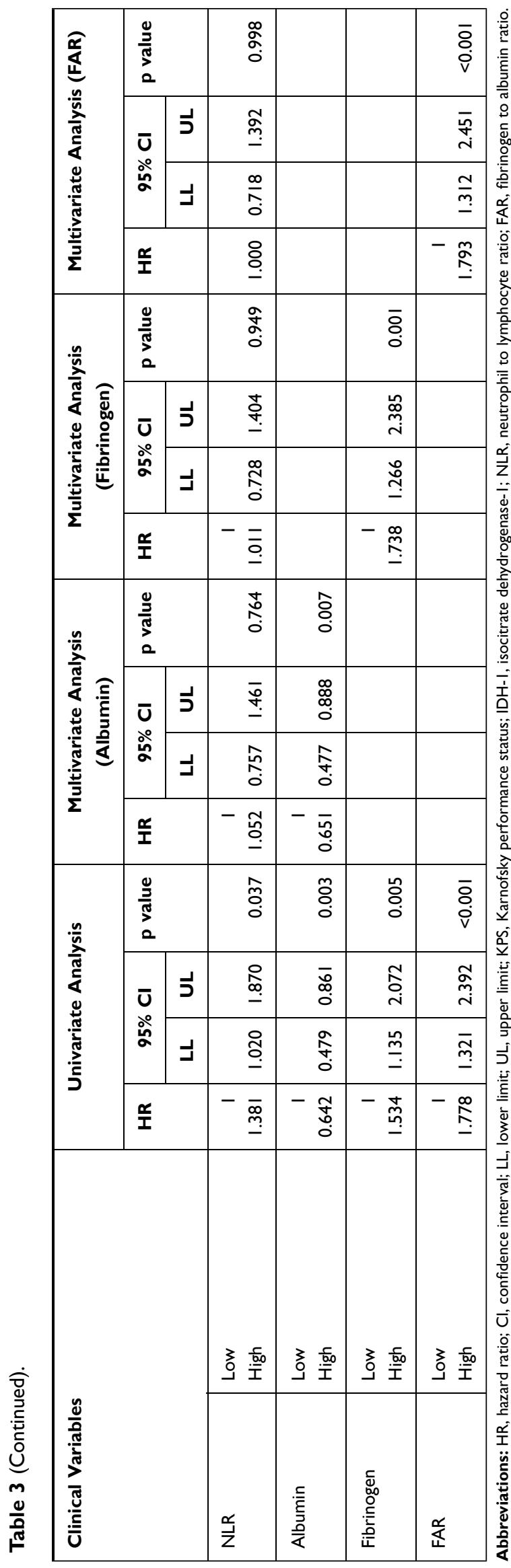

comprehensive search. In the research, $\mathrm{He}$ et al find higher FAR is related to shorter OS (Log rank test, $\mathrm{p}<0.001$ ), but the result is not further verified in multivariate analysis. $^{20}$

Fibrinogen and albumin are separately used to be prognostic indicators in various cancers. In general, low fibrinogen and high albumin concentration predict better prognosis. ${ }^{21-24}$ Usually, fibrinogen predicts prognosis of glioma patients combined with other hematological markers, such as fibrinogen-NLR score, fibrinogen-NLR-AGR score, fibrinogen-albumin score and Sanbo Scoring System. ${ }^{25-28}$ The above-mentioned prognostic models are relatively steady and effective based on analytic results.

At the same time, we have compared the prognostic predictive ability among albumin, fibrinogen, and FAR. For this purpose, three prognostic models are established based on significant prognostic variables from multivariate Cox regression. The result indicates that FAR is superior to fibrinogen and albumin in predicting prognosis.

The theory was firstly presented in 1865 that cancer favored the activation of blood coagulation with the appearance of hypercoagulable state or chronic disseminated intravascular coagulation (DIC) in these patients. ${ }^{6}$ Abnormalities in hemostatic parameters are present in $50 \%$ of patients with tumor and $95 \%$ of those with metastases. Coagulation activation and tumor progression were closely related. ${ }^{29}$ Different components of hemostatic system, including thrombin, tissue factor, FVIIa, FXa, fibrinogen, and vascular cells, are reported involving in process of neoangiogenesis and metastasis by both in vitro and in vivo tumor models. ${ }^{6,30,31}$ The synergistic effect between coagulation and inflammation further changes the tumor microenvironment and promotes progression and dissemination of tumors. ${ }^{32}$ This might explain why fibrinogen could serve as a prognostic indicator in malignancies. Similarly, coagulation indicators including d-dimer, prothrombin time (PT), and activated partial thromboplastin time (APTT) are also determined to have ability in predicting prognosis in various tumors. ${ }^{33-36}$

Serum albumin concentration, whose synthesis rate is affected by both nutrition and inflammation, can easily reflect status of diseases ${ }^{37}$ and it is usually reduced in tumors, as kind of wasting disease in company with microenvironmental inflammation. Therefore, it is not difficult to understand why albumin can serve as a predictor for tumor prognosis and progression. In the meta-analysis of Liu and Wang, subgroup analysis indicate that albumin is a significant prognostic indicator for GBM patients 
Table 4 Prognostic Model of Fibrinogen, Albumin, and FAR for GBM Patients

\begin{tabular}{|c|c|c|c|c|c|c|c|c|c|c|c|c|c|}
\hline \multirow{3}{*}{\multicolumn{2}{|c|}{ Clinical Variables }} & \multicolumn{4}{|c|}{ Prognostic Model (Albumin) } & \multicolumn{4}{|c|}{$\begin{array}{c}\text { Prognostic Model } \\
\text { (Fibrinogen) }\end{array}$} & \multicolumn{4}{|c|}{ Prognostic Model (FAR) } \\
\hline & & \multirow{3}{*}{$\begin{array}{c}\text { HR } \\
\\
1 \\
0.593\end{array}$} & \multicolumn{2}{|c|}{$95 \% \mathrm{Cl}$} & \multirow{3}{*}{$\begin{array}{c}\text { P value } \\
0.001\end{array}$} & \multirow{3}{*}{$\begin{array}{c}\text { HR } \\
\\
1 \\
0.560\end{array}$} & \multicolumn{2}{|c|}{$95 \% \mathrm{Cl}$} & \multirow{3}{*}{$\begin{array}{l}\text { P value } \\
<0.001\end{array}$} & \multirow{3}{*}{$\begin{array}{c}\text { HR } \\
\\
1 \\
0.615\end{array}$} & \multicolumn{2}{|c|}{$95 \% \mathrm{Cl}$} & \multirow{3}{*}{$\begin{array}{c}\text { P value } \\
0.003\end{array}$} \\
\hline & & & LL & UL & & & LL & UL & & & LL & UL & \\
\hline Gender & $\begin{array}{l}\text { Male } \\
\text { Female }\end{array}$ & & 0.430 & 0.818 & & & 0.405 & 0.776 & & & 0.445 & 0.850 & \\
\hline $\begin{array}{l}\text { Adjuvant } \\
\text { therapy }\end{array}$ & $\begin{array}{l}\text { Chemotherapy and } \\
\text { radiotherapy } \\
\text { Chemotherapy or } \\
\text { radiotherapy } \\
\text { None }\end{array}$ & $\begin{array}{c}1 \\
2.717 \\
4.017\end{array}$ & $\begin{array}{l}1.845 \\
2.743\end{array}$ & $\begin{array}{l}4.001 \\
5.881\end{array}$ & $\begin{array}{l}<0.00 \mathrm{I} \\
<0.00 \mathrm{I}\end{array}$ & $\begin{array}{c}1 \\
2.716 \\
4.845\end{array}$ & $\begin{array}{l}1.843 \\
3.243\end{array}$ & $\begin{array}{l}4.004 \\
7.238\end{array}$ & $\begin{array}{l}<0.001 \\
<0.001\end{array}$ & $\begin{array}{c}1 \\
2.699 \\
4.577\end{array}$ & $\begin{array}{l}1.832 \\
3.090\end{array}$ & $\begin{array}{l}3.977 \\
6.778\end{array}$ & $\begin{array}{l}<0.001 \\
<0.001\end{array}$ \\
\hline Ki67 & $\begin{array}{l}\geq 30 \% \\
<30 \%\end{array}$ & $\begin{array}{c}1 \\
0.653\end{array}$ & 0.480 & 0.889 & 0.007 & $\begin{array}{c}1 \\
0.638\end{array}$ & 0.468 & 0.869 & 0.004 & $\begin{array}{c}1 \\
0.623\end{array}$ & 0.457 & 0.850 & 0.003 \\
\hline IDH-I & $\begin{array}{l}\text { Mutant } \\
\text { Wildtype }\end{array}$ & $\begin{array}{c}1 \\
2.569\end{array}$ & 1.520 & 4.342 & $<0.001$ & $\begin{array}{c}1 \\
2.575\end{array}$ & 1.527 & 4.345 & $<0.001$ & $\begin{array}{c}1 \\
2.380\end{array}$ & 1.415 & 4.002 & 0.001 \\
\hline Albumin & $\begin{array}{l}\text { Low } \\
\text { High }\end{array}$ & $\begin{array}{c}1 \\
0.599\end{array}$ & $0.44 I$ & 0.813 & 0.001 & & & & & & & & \\
\hline Fibrinogen & $\begin{array}{l}\text { Low } \\
\text { High }\end{array}$ & & & & & $\begin{array}{c}1 \\
1.890\end{array}$ & 1.382 & 2.587 & $<0.001$ & & & & \\
\hline FAR & $\begin{array}{l}\text { Low } \\
\text { High }\end{array}$ & & & & & & & & & $\begin{array}{c}1 \\
1.925\end{array}$ & 1.416 & 2.618 & $<0.001$ \\
\hline C-index & & & & 711 & & & & 712 & & & & 714 & \\
\hline AIC & & & & 3.814 & & & & 6.516 & & & & 6.170 & \\
\hline
\end{tabular}

Abbreviations: HR, hazard ratio; Cl, confidence interval; LL, lower limit; UL, upper limit; IDH-I, isocitrate dehydrogenase-I; NLR, neutrophil to lymphocyte ratio; FAR, fibrinogen to albumin ratio.

$(\mathrm{HR}=0.95,95 \% \mathrm{CI}: 0.91-0.99, \mathrm{p}=0.018)$, especially in multivariate analysis. ${ }^{38}$

Unlike pathological biomarkers including IDH-1, ki67, methylation of O6-methylguanine-DNA methyltransferase (MGMT), detection of hematological markers is convenient, affordable, and relatively innocuous. And in general, most of these markers are contained in regular preoperative examinations. Combined with other hematological prognostic markers such as NLR, PLR, and AGR, even the constructed prognostic models based on these markers, we can evaluate prognosis of GBM in more accurate ways. And the potential mechanism is needed to be elucidated.

There are still some limitations in our study. At first, FAR is absent in the postoperative follow-up plan, so we cannot know its changes and its relationship with prognosis which could help us to further evaluate its clinical significance throughout the whole course of disease. Secondly, the sample size is relatively small so that we did not build a validation group to further verified the result. Thirdly, it is difficult to accurately and timely assess the progression of GBM after operation considering its early recurrence and rapid progression, so we did not include clinical data on disease progression like progression-free survival (PFS). Last but not least, patients with recurrent GBM who receive operation in the same time period should be included to enrich the research.

\section{Conclusion}

To our knowledge, this is the first study focusing on validating the clinical significance of preoperative FAR in GBM. In the current study, FAR is not only significantly associated with clinical characteristics such as age, preoperative KPS, IDH-1 mutation status, condition of preoperative seizures, but also determined to be an independent 
risk factor of prognosis in patients with newly-diagnosed GBM. And the prognostic predictive ability of FAR is stronger than fibrinogen and albumin.

\section{Acknowledgment}

We would like to express our gratitude to all the colleagues who have assisted clinical data collection. And we also express our gratitude to Professor Liu Yanhui, who has participated in the design of the study and supervised the procedure of the current research.

\section{Funding}

This study is supported by National Natural Science Foundation of China (Grant No.81802096).

\section{Disclosure}

The authors declare no conflicts of interest.

\section{References}

1. Vartanian A, Singh SK, Agnihotri S, et al. GBM's multifaceted landscape: highlighting regional and microenvironmental heterogeneity. Neuro Oncol. 2014;16(9):1167-1175. doi:10.1093/neuonc/nou035

2. Shergalis A, Bankhead A 3rd, Luesakul U, Muangsin N, Neamati N. Current challenges and opportunities in treating glioblastoma. Pharmacol Rev. 2018;70(3):412-445. doi:10.1124/pr.117.014944

3. Pearson JRD, Regad T. Targeting cellular pathways in glioblastoma multiforme. Signal Transd Targeted Ther. 2017;2:17040. doi:10.1038/sigtrans.2017.40

4. Wesseling P, Capper D. WHO 2016 classification of gliomas. Neuropathol Appl Neurobiol. 2018;44(2):139-150. doi:10.1111/ nan.12432

5. Louis DN, Perry A, Reifenberger G, et al. The 2016 World Health Organization classification of tumors of the central nervous system: a summary. Acta Neuropathol. 2016;131(6):803-820. doi:10.1007/ s00401-016-1545-1

6. Falanga A, Marchetti M, Vignoli A. Coagulation and cancer: biological and clinical aspects. J Thrombosis Haemostasis. 2013;11 (2):223-233. doi:10.1111/jth.12075

7. Cai HX, Li XQ, Wang SF. Prognostic value of fibrinogen and D-dimer-fibrinogen ratio in resectable gastrointestinal stromal tumors. World J Gastroenterol. 2018;24(44):5046-5056. doi:10.3748/wjg.v24.i44.5046

8. Liu R, Zhou X, Zou L, et al. Clinicopathological and prognostic significance of preoperative plasma fibrinogen level in patients with upper urinary tract urothelial carcinoma: a retrospective tumor marker prognostic study. Int J Surg. 2019;65:88-93. doi:10.1016/j. ijsu.2019.03.022

9. Wang H, Gao J, Bai M, et al. The pretreatment platelet and plasma fibrinogen level correlate with tumor progression and metastasis in patients with pancreatic cancer. Platelets. 2014;25(5):382-387. doi:10.3109/09537104.2013.827782

10. Polterauer S, Grimm C, Seebacher V, et al. Plasma fibrinogen levels and prognosis in patients with ovarian cancer: a multicenter study. The Oncologist. 2009;14(10):979-985. doi:10.1634/theoncologist.2009-0079

11. Hülshoff A, Schricker T, Elgendy H, Hatzakorzian R, Lattermann R. Albumin synthesis in surgical patients. Nutrition (Burbank, Los Angeles County, Calif). 2013;29(5):703-707. doi:10.1016/j.nut.2012.10.014
12. Garcia-Martinez R, Caraceni P, Bernardi M, Gines P, Arroyo V, Jalan R. Albumin: pathophysiologic basis of its role in the treatment of cirrhosis and its complications. Hepatology (Baltimore, Md). 2013;58(5):1836-1846. doi:10.1002/hep.26338

13. Al-Shaiba R, McMillan DC, Angerson WJ, Leen E, McArdle CS, Horgan P. The relationship between hypoalbuminaemia, tumour volume and the systemic inflammatory response in patients with colorectal liver metastases. Br J Cancer. 2004;91(2):205-207. doi:10.1038/sj.bjc. 6601886

14. Dupré A, Malik HZ. Inflammation and cancer: what a surgical oncologist should know. Eur J Surg Oncol. 2018;44(5):566-570.

15. Wang YY, Liu ZZ, Xu D, Liu M, Wang K, Xing BC. FibrinogenAlbumin Ratio Index (FARI): a more promising inflammation-based prognostic marker for patients undergoing hepatectomy for colorectal liver metastases. Ann Surg Oncol. 2019;26(11):3682-3692. doi:10.1245/s10434-019-07586-3

16. Xu Q, Yan Y, Gu S, et al. A novel inflammation-based prognostic score: the fibrinogen/albumin ratio predicts prognoses of patients after curative resection for hepatocellular carcinoma. J Immunol Res. 2018;2018:4925498.

17. Li SQ, Jiang YH, Lin J, et al. Albumin-to-fibrinogen ratio as a promising biomarker to predict clinical outcome of non-small cell lung cancer individuals.. 2018;7(4):1221-1231.

18. Sun F, Peng HX, Gao QF, et al. Preoperative circulating FPR and CCF score are promising biomarkers for predicting clinical outcome of stage II-III colorectal cancer patients. Cancer Manag Res. 2018;10:2151-2161. doi:10.2147/CMAR.S167398

19. Du JH, Lu J. Circulating CEA-dNLR score predicts clinical outcome of metastatic gallbladder cancer patient. J Clin Lab Anal. 2019;33(2): e22684.

20. He ZQ, Duan H, Lin FH, et al. Pretreatment neutrophil-tolymphocyte ratio plus albumin-to-gamma-glutamyl transferase ratio predict the diagnosis of grade III glioma. Ann Transl Med. 2019;7 (22):623. doi:10.21037/atm.2019.11.24

21. Lu J, Chen S, Li X, et al. Gastrointestinal stromal tumors: fibrinogen levels are associated with prognosis of patients as blood-based biomarker. Medicine. 2018;97(17):e0568. doi:10.1097/MD.0000000000010568

22. Luo Y, Kim HS. Elevated plasma fibrinogen levels and prognosis of epithelial ovarian cancer: a cohort study and meta-analysis. May. 2017;28(3):e36.

23. Seebacher V, Grimm C, Reinthaller A, et al. The value of serum albumin as a novel independent marker for prognosis in patients with endometrial cancer. Eur J Obstet Gynecol Reprod Biol. 2013;171 (1):101-106. doi:10.1016/j.ejogrb.2013.07.044

24. Kao HK, Löfstrand J, Loh CY, et al. Nomogram based on albumin and neutrophil-to-lymphocyte ratio for predicting the prognosis of patients with oral cavity squamous cell carcinoma. Sci Rep. 2018;8 (1):13081. doi:10.1038/s41598-018-31498-Z

25. Wu Y, Song Z, Sun K, Rong S. A novel scoring system based on peripheral blood test in predicting grade and prognosis of patients with glioma. OncoTargets Ther. 2019;12:11413-11423. doi:10.2147/ OTT.S236598

26. Hao Y, Li X, Chen H, et al. A cumulative score based on preoperative neutrophil-lymphocyte ratio and fibrinogen in predicting overall survival of patients with glioblastoma multiforme. World Neurosurg. 2019;128:e427-e433. doi:10.1016/j.wneu.2019.04.169

27. Wang PF, Zhang J, Cai HQ, et al. Sanbo scoring system, based on age and pre-treatment hematological markers, is a non-invasive and independent prognostic predictor for patients with primary glioblastomas: a retrospective multicenter study. J Cancer. 2019;10(23):5654-5660. doi:10.7150/jca.33047

28. He ZQ, Duan H, Ke C, et al. Evaluation of cumulative prognostic score based on pretreatment plasma fibrinogen and serum albumin levels in patients with newly diagnosed high-grade gliomas. Oncotarget. 2017;8 (30):49605-49614. doi:10.18632/oncotarget.17849 
29. Iqbal S. Role of thrombomodulin in cancer biology. Breast (Edinburgh, Scotland). 2000;9(5):264-266. doi:10.1054/ brst.2000.0186

30. Xie WZ, Leibl M, Clark MR, Dohrmann P, Kunze T, Gieseler F. Activation of the coagulation system in cancerogenesis and metastasation. Biomed Pharmacother/Biomedecine \& Pharmacotherapie. 2005;59(3):70-75. doi:10.1016/j.biopha.2005.01.001

31. Goad KE, Gralnick HR. Coagulation disorders in cancer. Hematol Oncol Clin North Am. 1996;10(2):457-484.

32. Iyengar NM, Gucalp A, Dannenberg AJ, Hudis CA. Obesity and cancer mechanisms: tumor microenvironment and inflammation. J Clin Oncol. 2016;34(35):4270-4276. doi:10.1200/JCO.2016.67.4283

33. Hoke M, Dieckmann K, Koppensteiner R, Schillinger M, Marosi C, Mlekusch W. Prognostic value of plasma d-dimer levels in patients with glioblastoma multiforme - Results from a pilot study. Wien Klin Wochenschr. 2011;123(7-8):199-203. doi:10.1007/s00508-011-1556-9

34. Wang HS, Ge XX, Li QP, Nie JJ, Miao L. Clinical significance of prothrombin time in cholangiocarcinoma patients with surgeries. 2019;2019:3413969.
35. Teng HW, Chen PM, Yang YH, Gau JP. The prolonged activated partial thromboplastin time at diagnosis indicates less favorable prognosis in IgA myeloma. Jpn J Clin Oncol. 2007;37(8):609-614. doi:10.1093/jjco/hym074

36. Watanabe A, Araki K, Harimoto N, et al. D-dimer predicts postoperative recurrence and prognosis in patients with liver metastasis of colorectal cancer. Int J Clin Oncol. 2018;23(4):689-697. doi:10.1007/s10147-018-1271-x

37. Don BR, Kaysen G. Serum albumin: relationship to inflammation and nutrition. Semin Dial. 2004;17(6):432-437. doi:10.1111/j.08940959.2004.17603.x

38. Liu M, Wang L. Prognostic significance of preoperative serum albumin, albumin-to-globulin ratio, and prognostic nutritional index for patients with glioma: a meta-analysis. Medicine. 2020;99(27): e20927. doi:10.1097/MD.0000000000020927

\section{Publish your work in this journal}

Cancer Management and Research is an international, peer-reviewed open access journal focusing on cancer research and the optimal use of preventative and integrated treatment interventions to achieve improved outcomes, enhanced survival and quality of life for the cancer patient.
The manuscript management system is completely online and includes a very quick and fair peer-review system, which is all easy to use. Visit http://www.dovepress.com/testimonials.php to read real quotes from published authors. 